\title{
Etnografie Sonore / Sound Ethnographies
}

Edizioni Museo Pasqualino, Palermo (versione online) - Neoclassica SRL, Roma (versione a stampa), 2018

Gioele Zisa

\section{(2) OpenEdition}

\section{Journals}

Edizione digitale

URL: http://journals.openedition.org/aam/763

ISSN: 2038-3215

\section{Editore}

Dipartimento Culture e Società - Università di Palermo

\section{Notizia bibliografica digitale}

Gioele Zisa, "Etnografie Sonore / Sound Ethnographies », Archivio antropologico mediterraneo [Online], Anno XXI, n. 20 (2) | 2018, online dal 31 décembre 2018, consultato il 23 septembre 2020. URL : http:// journals.openedition.org/aam/763

Questo documento è stato generato automaticamente il 23 settembre 2020.

\section{(c) (i) (9)}

Archivio antropologico mediterraneo è distribuita con Licenza Creative Commons Attribuzione - Non commerciale - Non opere derivate 4.0 Internazionale. 


\section{Etnografie Sonore / Sound Ethnographies}

Edizioni Museo Pasqualino, Palermo (versione online) - Neoclassica SRL, Roma (versione a stampa), 2018

Gioele Zisa

NOTIZIA

Etnografie Sonore / Sound Ethnographies, anno I, numero 1, Edizioni Museo Pasqualino, Palermo (versione online) - Neoclassica SRL, Roma (versione a stampa), 2018.

ISNN 2611-4267 
1 Nonostante nell'ultimo ventennio si sia assistito in Italia a un incremento significativo delle ricerche sulle musiche di tradizione orale e l'Etnomusicologia svolga un ruolo sempre più importante nell'accademia e in varie istituzioni pubbliche e private, si avvertiva l'assenza di una rivista italiana in questo settore. Troppi anni sono trascorsi dalla pubblicazione di Culture musicali. Quaderni di etnomusicologia (1982-1990), promossa da Diego Carpitella con la collaborazione della prima generazione dei suoi allievi, e di EM (1993-2000, 2003-2006), sostenuta dall'Accademia Nazionale di Santa Cecilia. Questa nuova rivista giunge pertanto a colmare un vuoto fortemente avvertito, grazie all'iniziativa dei tre codirettori,

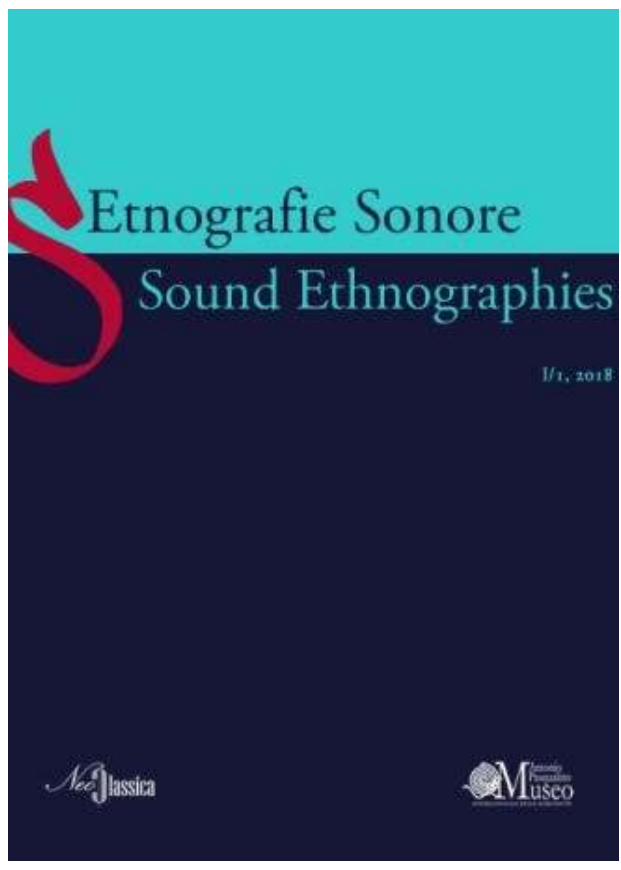
Giorgio Adamo (Università di Roma “Tor

Vergata"), Sergio Bonanzinga (Università di Palermo) e Nico Staiti (Università di Bologna), insieme al direttore responsabile Rosario Perricone, in felice sinergia con le Edizioni Museo Pasqualino (Palermo), per la versione online corredata da contenuti multimediali, e le Edizioni NeoClassica (Roma), per la versione a stampa, e con il patrocinio delle tre Università d'appartenenza dei direttori.

2 Come si legge sulla quarta di copertina, Etnografie Sonore / Sound Ethnographies è un semestrale peer reviewed che pubblica contributi in italiano e in inglese. Il nuovo progetto editoriale «nasce dall'esperienza di ricerca dell'etnomusicologia italiana e si propone come terreno di incontro in ambito internazionale per tutte le prospettive di indagine che affrontano la complessità e la trasformazione delle pratiche musicali». Come inoltre ricordano i direttori, l'esperienza di ricerca dell'etnomusicologia italiana ha sempre manifestato dei tratti distintivi, noti a livello internazionale. Tra questi, l'equilibrio tra l'adozione del proprio paese come terreno di indagine e l'interesse rivolto a contesti culturali altri; l'importanza di impiegare gli approcci teorici e metodologici dell'antropologia culturale, soffermandosi in particolare sugli aspetti sociali dei fenomeni musicali, coinvolgendone i protagonisti; la necessità di un dialogo con la musicologia storica, di un interesse agli approcci linguistici e letterari al testo dei canti, e di un'analisi antropologica in una prospettiva semiotico-strutturalista; l'importanza di combinare alla ricerca sul campo l'analisi storica per una migliore comprensione dell'universo musicale indagato; l'attenzione alla marginalità sociale e culturale, ai fenomeni connessi all'emigrazione e alla diaspora; l'interesse nei confronti della canzone d'autore, della musica popolaresca urbana e dei nuovi linguaggi e generi musicali. Elemento innovativo e centrale è l'integrazione della riflessione critica scritta con la riproduzione sonora e visiva, grazie alle possibilità offerte dalla multimedialità su internet. Per questa ragione la rivista è pubblicata anche online, offrendo la possibilità di corredare i testi con materiali multimediali (suoni e filmati).

3 La rivista si avvale di un comitato scientifico costituito da nomi celebri dell'etnomusicologica italiana e internazionale, nonché da un comitato di consulenti 
internazionale e una redazione formata dalle nuove generazioni di giovani ricercatori, italiani e non. Uno spazio significativo, infatti, come emerge dal primo numero della rivista, è stato dato a giovani studiosi italiani, i quali hanno proposto i risultati delle loro indagini sul campo. Oltre agli articoli, è presente una sezione dal titolo "Saggi audiovisivi" dove non sarà presentato un testo scritto con eventuali contributi video allegati, quanto piuttosto un prodotto filmico autonomo, accompagnato da una presentazione in forma scritta dell'autore sottoposta a peer-review. Si tratta della prima occasione di presentare questa nuova modalità di comunicazione scientifica. A questo spazio all'interno della rivista si aggiunge anche la sezione "Interventi", in cui si propongono riflessioni, opinioni e considerazioni di politica culturale, non necessariamente condivise dalla direzione e dalla redazione, utili a stimolare il dibattito all'interno della disciplina. In questo numero è presente il contributo polemico di Nico Staiti dal titolo “The 'Rose Garden': Against Racism in Ethnomusicology" sul tema del razzismo, centrale nella riflessione etnomusicologica, in risposta al saggio dello studioso rumeno Marin Marian-Balasa intitolato "Romany Music and Gypsy Criminality" (2004). La sezione "Istituzioni", invece, è dedicata a musei, archivi, centri di ricerca che presentano sé stessi e le proprie attività. In questo numero il Museo internazionale delle marionette A. Pasqualino di Palermo, ente che sostiene e promuove la rivista, è presentato dal suo direttore Rosario Perricone, mentre il Phonogrammarchiv - Österreichische Akademie der Wissenschaften, il primo archivio sonoro al mondo fondato nel 1899, da Gerda Lechleitner.

Il primo numero della rivista si apre con il contributo del noto etnomusicologo austriaco Gerhard Kubik dal titolo "Culture Contact: Cognitive and Psychodynamic Aspects. Transcultural Understanding in Art History, Religion, Music and Animation" in cui le dinamiche del culture contact vengono analizzate, grazie agli apporti teoricometodologici dell'antropologia culturale e della psicoanalisi. L'autore parte dalla ormai ovvia costatazione che due persone che si incontrano e interagiscono tra loro, pur parlando lingue diverse che condividono con milioni di altri individui, non possono certo essere ritenute rappresentanti di una cultura, mostrando ognuno al contrario una sua specifica "identità culturale". Ognuno di noi sviluppa, infatti, profili che integrano ciò che abbiamo assimilato consapevolmente e inconsciamente dall'ambiente in cui abbiamo vissuto. Si tratta di ciò che Kubik definisce "profilo culturale individuale". È il profilo culturale di un individuo che determina la natura delle sue reazioni in una situazione di contatto interculturale. I profili culturali individuali sono pertanto complessi nei contenuti, transitori e mutevoli. Al contatto con gli altri, il profilo culturale di un individuo non è espresso in toto, ma sempre in risposta a specifiche aspettative della persona con cui si sta interagendo. L'autore fa preciso riferimento alla teoria delle "identità multiple", e in particolare agli studi della psicologa vietnamita Julia Ha, ma anche di Carl G. Jung e George Devereux.

5 Nello studio psicologico del contatto culturale emergono due livelli paralleli di indagine: il livello cognitivo e quello psicodinamico. Per il primo livello, Kubik fa preciso riferimento ai lavori di Melville J. Herskovits in Nord America, Caraibi e Sud America e al concetto da lui elaborato di reinterpretazione, secondo il quale, in un incontro interculturale, comprendiamo qualcosa di nuovo e non familiare collocandolo all'interno delle categorie formulate dalle nostre lingue. Tuttavia, sottolinea l'autore, sebbene ogni attore dell'incontro interculturale interpreti i fenomeni altri sulla base delle proprie categorie, è possibile rintracciare in alcuni campi, come le belle arti e l'animazione cinematografica, ciò che egli definisce un ponte cognitivo. Come dimostrano 
i risultati del test da lui condotto CCCT (Cross-Cultural Comprehension Test) in Brasile nel 1974, che consiste nel sottoporre a una persona un breve filmato, della durata di 3-5 minuti, appartenente a una cultura diversa e lasciare che scriva le sue osservazioni, nel contatto culturale c'è sempre un'area minima di comprensione che va oltre le barriere culturali, e ciò è particolarmente evidente con i fenomeni musicali e artistici. Il secondo livello di indagine è quello psicodinamico riguardante i processi inconsci. Secondo Kubik un'altra cultura esercita sempre una spinta sull'inconscio di un individuo. Ciò è dovuto al fatto che l'altra cultura rappresenta un'alternativa ai modi di vivere propri di una persona. Attraverso le lenti della psicoanalisi l'autore discute alcuni concetti quali quello di transfert, della reazione cosiddetta apartheid e dello shock culturale. L'obiettivo del contribuito, dunque, è riassumere la esperienza cinquantennale sul campo dell'autore entro le cornici di una teoria transculturalmente valida del contatto culturale e delle sue basi psicologiche.

6 Segue l'articolo di Razia Sultanova dal titolo "Female Teachers: a New Concept of Traditional Master-Apprentice Knowledge Transmission in Central Asian Music (Ferghana Valley Case)" in cui l'etnomusicologa mostra l'importanza, finora poco studiata e documentata, del ruolo delle donne nella trasmissione del sapere musicale nella Valle di Fergana, nella parte orientale dell'Uzbekistan, lungo l'antica Via della Seta. Grazie a un approccio interdisciplinare, che coniuga indagine etnomusicologica e studi di genere, l'autrice dimostra quanto le donne in questa valle, nota per la sua importanza storico-culturale, abbiano preservato durante il periodo sovietico i generi musicali tradizionali, popolari e religiosi. È importante ricordare che per secoli in Uzbekistan, come in altri paesi musulmani dell'Asia centrale, la separazione tra uomini e donne è stata netta. Questo è testimoniato anche dalla struttura architettonica delle abitazioni, divise in due parti separate: tashqari, la parte esterna, maschile e ichkari, quella interna, femminile. Ciò ha permesso la trasmissione di generi musicali, religiosi e non, all'interno di contesti esclusivamente femminili, finora poco documentati. Per esempio, l'autrice dimostra, attingendo a una varietà di fonti letterarie, figurative e fotografiche, il ruolo storico delle donne della Valle di Fergana nel suonare il dutar, il tradizionale liuto a due corde a manico lungo diffuso in Iran come in Asia centrale, così come il loro contributo al genere musicale uzbeko shashmaqam.

7 Sultanova si sofferma, inoltre, sulla trasmissione maestro-apprendista in tali contesti femminili. L'otin-oy è una delle forme principali di tale relazione, che ha permesso la trasmissione per generazioni del canto spirituale sufi e della conoscenza religiosa in cui la recitazione musicale è alla base della performance. Le otin-oys sono donne non istruite, che tuttavia posseggono una conoscenza spirituale e religiosa, accessibile solo a persone scelte. Soppresse durante il periodo sovietico, rimasero tuttavia attive clandestinamente per quasi settant'anni. Si tratta di vedove o madri di bambini disabili che giocano un ruolo importante in tutti i riti di passaggio, come matrimoni e funerali, così come durante le festività islamiche $\mathrm{e}$ i cui rituali integrano elementi di altri antichi saperi religiosi, come lo zoroastrismo, il tengrianismo e il manicheismo. La studiosa conclude sottolineando la necessità di un supporto per la tutela del patrimonio musicale tradizionale uzbeko e di una maggiore consapevolezza del ruolo della donna nello sviluppo culturale del paese.

8 Lorenzo Vanelli propone nel suo contributo "Between the Blues and Africa: Transformations of Narratives About African American Hollers" i primi risultati di una ricerca in corso sui field hollers, un genere di musica vocale afro-americana eseguito nel 
sud degli Stati Uniti fino agli anni Sessanta del secolo scorso, in un contesto sociale altamente razzista, come mezzo di comunicazione, in particolare nei penitenziari statali e nei campi per la costruzione degli argini dei fiumi. L'articolo riassume le principali teorie sugli hollers, in particolare sulla loro connessione con il blues e alcune tradizioni musicali africane. Come sottolinea Vanelli, questo tipo di relazioni, tuttavia, devono essere accertate e documentate con maggiore precisione.

Nel suo interessante articolo "New Urban Soundscapes: Angklung Buskers in Yogyakarta" Daniele Zappatore analizza, attraverso una ricerca etnografica, un affascinante esempio di musica urbana di recentissimo sviluppo, noto come angklung buskers, a Yogyakarta, nell'isola di Giava in Indonesia. Si tratta di gruppi di musicisti di strada che si guadagnano da vivere esibendosi quotidianamente negli spazi più trafficati della città. Lo studioso si sofferma sul modo in cui questa produzione musicale si integra con il paesaggio sonoro della moderna metropoli. Come mostra Zappatore, il fenomeno degli angklung buskers è connesso alle migrazioni interne, dalla zona rurale di Kabupaten Banyumas alla metropoli, di questi musicisti di strada, pengamen, i quali hanno portato con sé una specifica pratica musicale ed il loro ensemble di strumenti autoctoni tradizionali, chiamato kentongan (o tek-tek). Dal 2008 il fenomeno si è diffuso rapidamente a Yogyakarta, riscontrando un grande successo di pubblico e incoraggiando molti giovani a unirsi agli ensemble già esistenti o a crearne di nuovi. L'autore si sofferma inoltre sulle politiche governative in merito alla regolazione delle bande angklung, vista l'alta competizione tra musicisti di strada per occupare i luoghi più affollati della città. Zappatore, inoltre, ne documenta l'attività - si notino gli esempi video al testo correlati - ponendo attenzione alle storie di vita di questi giovani musicisti, alle tecniche esecutive, ai luoghi delle performance e alla organologia (lo strumento principale è l'idiofono angklung che dà il nome alla banda). Il repertorio è vario e aperto a una vasta gamma di forme musicali diverse, in grado di soddisfare $i$ gusti di un pubblico in continua evoluzione, in particolare brani di musica indonesiana contemporanea, appresi dagli angklung pengamen in Internet, loro fonte di ispirazione, e da loro semplificati. L'elemento importante, dunque, che caratterizza tale fenomeno musicale è ciò che l'autore definisce "interpretazione creativa": queste bande di musicisti infatti combinano strumenti di origine "tradizionale" con le esigenze di produzione della musica "globale", riproponendo nel loro stile personale le canzoni di maggior successo indonesiane e non solo.

Emanuele Tumminello in "Una indagine sulla musica di tradizione orale in Alto Svaneti (Georgia)" presenta i risultati della sua ricerca etnografica condotta nel 2016 in quattro villaggi dell'Alto Svanati in Georgia. La ricerca si concentra sui riti e cerimonie festive in cui la musica gioca un ruolo fondamentale. Il repertorio musicale svano è costituito principalmente da inni sacri e balli cantati (perxuli). I primi seguono le modalità consuete del canto svano, dall'andamento prevalentemente sillabico, basato sulla sovrapposizione di tre voci: alta, media e bassa. I secondi sono eseguiti in particolari giorni di festa, all'interno o all'esterno delle abitazioni, o presso luoghi di culto, e le tematiche prevalenti nei loro testi poetici riguardano la caccia, le battaglie, la devozione e la vita quotidiana. Esecuzioni musicali possono svolgersi anche in occasione dei supra, banchetti tradizionali contraddistinti dalla grande abbondanza di cibo, connessi a celebrazioni festive, ma anche funebri. I testi poetici dei canti sono oralmente trasmessi in "svano", l'antica lingua locale. In occasione dei riti funebri gli uomini eseguono lo zär, inno funebre composto esclusivamente da esclamazioni di dolore e disperazione, all'esterno dell'abitazione del defunto, nel tragitto verso il 
cimitero e durante la sepoltura. Le donne eseguono invece specifici lamenti (lilččal) all'interno dell'abitazione, intorno alla salma. Tumminello documenta anche la costruzione e l'uso dei due unici strumenti tradizionali svani tuttora impiegati: il čangi, arpa angolare in legno a otto corde e il čuniri, fidula realizzata in legno e pelle di capra, munita di tre corde poste in vibrazione mediante l'impiego di un archetto. Il testo è correlato da un ricco apparato di testi verbali, trascrizioni musicali, fotografie e registrazioni video.

11 Giuseppina Colicci nel suo contributo "Sicilia 1955. La Raccolta 27 degli Archivi di Etnomusicologia (Roma)" ricostruisce la genesi e l'ideazione dell'ambizioso progetto della Raccolta 27 del Centro Nazionale Studi di Musica Popolare di Roma (CNSMP), oggi Archivi di Etnomusicologia (AE). Si tratta di una importante ricerca sul campo in parte finanziata dalla Regione Siciliana attraverso l'allora nascente Centro Internazionale Studi Musiche Mediterranee (CISMMED). Durante la spedizione, che coinvolse studiosi italiani e stranieri, furono registrati 194 brani nelle province di Agrigento, Caltanissetta e Siracusa. Attraverso documenti inediti del Musée des Art et Traditions Populaires di Parigi, del Musée royal de l'Afrique centrale di Tervuren (Belgio) e dell'Accademia Nazionale di Santa Cecilia di Roma, l'autrice ricostruisce e descrive questo importante lavoro sul campo, condotto in un momento in cui si formava l'etnomusicologia italiana anche attraverso contatti e collaborazioni con l'ambiente scientifico internazionale. Colicci fornisce infine una rassegna relativa agli esiti editoriali della Raccolta, ponendo particolare attenzione al lavoro dello studioso belga Paul Collaer che, a causa della scomparsa del siciliano Ottavio Tiby, assunse l'incarico di sistemare e analizzare tali materiali sonori.

Per la sezione "Saggi audiovisivi", è pubblicato il cortometraggio esperienziale "A Common Place" (10 min.) di Jennie Gubner, tratto dalla sua tesi di dottorato, sul mondo del tango in un bar di Buenos Aires. Il film è corredato da un testo dal titolo "More than Fishnets \& Fedoras: Filming Social Aesthetics in the Neighborhood Tango Scenes of Buenos Aires \& The Making of A Common Place (2010)", in cui l'autrice descrive i presupposti teorici e gli intenti che stanno alla base della produzione filmica. Come sottolinea Gubner, il tango argentino è stato oggigiorno sottoposto a un processo di "ipersessualizzazione da bordello" per seguire le direttrici dell'industria turistica globale, dando vita a quello che in Argentina è definito "tango da esportazione". Tuttavia, negli ultimi dieci anni nuove generazioni di artisti di tango hanno contestato questi stereotipi, sottolineando la stretta relazione tra il fenomeno musicale e l'attuale vita quotidiana cittadina di Buenos Aires. Il tango è infatti in questi bar in primo luogo suonato e non danzato. Il luogo al centro dell'etnografia di Gubner è il piccolo bar "Lo de Roberto" (o "El Boliche de Roberto") nel barrio Almagro della capitale argentina. Si tratta di un luogo che a partire dagli anni ' 90 è diventato un popolare ritrovo notturno per la musica dal vivo del tango e ha svolto un ruolo importante nel trasformare il tango in una intima e partecipativa cultura di quartiere per le generazioni più giovani, anche al di fuori dell'Argentina. Il lavoro di Gubner è innovativo e stimolante, ed è interessante il modo in cui il film - che la stessa autrice definisce: «sensory filmmaking as an experiential (visual, aural, evocative) mode of knowledge production» - e il testo scritto si integrano a vicenda. 